\title{
Long Term Trends in the Frequencies of Given Names
}

\author{
Douglas A. Galbi \\ Federal Communications Commission
}

The frequency distributions of personal given names offer important insights into the nature of the information economy. Here I present data on the popularity of the most frequent personal given names in the United Kingdom over the past millennium. The data show that the popularity of names, like the popularity of other symbols and artifacts associated with the information economy, can be usefully viewed as a power law. The frequency distribution of personal names, graphed as the logarithm of name popularity against the logarithm of name popularity rank, is similar to other popularity distributions where people and organizations are free to create and choose among many collections of symbols used in a similar way. Naming is seen to be representative of more general patterns of behavior in the information economy. Furthermore, the data suggest that historically distinctive changes in the information economy occurred in conjunction with the Industrial Revolution.

\section{Names and the Information Economy}

An important component of the information economy consists of the production and use of symbols. Names are an important type of symbol and choosing a "good" name involves an assessment of the social valuation of the name. The frequency distribution of names provides evidence of their social valuation and can offer insights into the historical development of the information economy. The frequency distribution of a name indicates the number of people who share the experience of being called by that name.

Since the early 19th century, the distribution of personal given names in the UK has evolved differently that it did over the previous eight centuries. Simple indicators of this change are the trends seen in the relative frequency of the most popular name, the three most popular names, and the ten most popular names. These show practically no 
differences from about 1300 to 1800 . Since then all of these measures have changed dramatically, the result being a flattening in the name frequency distribution, viewed as a graph of the logarithm of name popularity against the logarithm of name popularity rank. This change in the evolution of the name frequency distribution early in the 19th century is suggestive of a more general change in the information economy about that time.

\section{Popularity of the Most Frequent Names}

Measuring name frequencies in actual samples requires attention to name definition and standardization. Given names can include multiple names and name variants as well as abbreviations, non-standard spellings, and likely mistakes in recording (e.g., William, Bill, Wm., Williamus). Unlike sampling variability, coding variability does not fall with sample size. Throughout the analysis here, names have been truncated to the shorter of either the first eight letters of the name or the letters preceding the first period, space, hyphen, or other non-alphabetic character (e.g. Wm. and Williamus would be truncated to $\mathrm{Wm}$ and William, respectively). These shortened names have then been standardized through a name coding process available on the internet (GINAP, by which, for example, Bill is standardized to William). ${ }^{1}$ This procedure attempts to identify feasibly and consistently names with common communicative properties. ${ }^{2}$ Experience with different name samples suggests that this procedure can reduce coding variability to less than half of one percent for the popularity of a single name and less than three percent for total popularity of the top ten names (Galbi 2001, Sec. I.B. and Appendix B).

Over the past two hundred years, the relative popularity of the most frequent names given in the UK has steadily declined. Table 1 shows popularity statistics for the most frequent names since 1800 . The data in this table come from census records, birth records, and doctor registrations, collected from the sources documented in detail in Galbi (2001). In the UK from 1800 to 1994, the frequency of the most popular female name fell from $23.9 \%$ to $3.4 \%$ and that of the most popular male name fell from $21.5 \%$ to $4.2 \%$. The popularity of the ten most frequent names for females fell from $82.0 \%$ to $23.8 \%$ and for male names from $51.5 \%$ to $28.4 \%$. 
Table 1. Frequencies of Most Popular UK Given Names, 1800-1994.

\begin{tabular}{llrrrllllr}
\hline & & & & & \multicolumn{5}{c}{ Males } \\
Birth & Top 1 & & Top 3 Top 10 & Top 1 & \multicolumn{2}{c}{ Top 3 Top 10 } \\
Year & Name & $\%$ & $\%$ & $\%$ & Name & $\%$ & $\%$ & $\%$ \\
1800 & Mary & 23.9 & 53.2 & 82.0 & John & 21.5 & 51.5 & 84.7 \\
1810 & Mary & 22.2 & 50.7 & 79.4 & John & 19.0 & 47.0 & 81.4 \\
1820 & Mary & 20.4 & 47.7 & 76.5 & John & 17.8 & 44.9 & 80.4 \\
1830 & Mary & 19.6 & 45.4 & 75.8 & John & 16.4 & 42.3 & 78.2 \\
1840 & Mary & 18.7 & 43.2 & 75.0 & William & 15.4 & 40.3 & 76.0 \\
1850 & Mary & 18.0 & 41.0 & 72.1 & William & 15.2 & 38.7 & 73.8 \\
1860 & Mary & 16.3 & 37.0 & 68.3 & William & 14.5 & 36.2 & 69.8 \\
1870 & Mary & 13.3 & 31.5 & 61.1 & William & 13.1 & 31.7 & 63.5 \\
1880 & Mary & 10.6 & 25.4 & 53.8 & William & 11.7 & 28.5 & 58.9 \\
1900 & Elizabet & 7.2 & 16.2 & 38.5 & William & 9.0 & 22.9 & 50.9 \\
1925 & Mary & 6.7 & 16.8 & 38.7 & John & 7.3 & 17.6 & 38.0 \\
1944 & Margaret & 4.5 & 12.6 & 31.7 & John & 8.3 & 20.7 & 39.9 \\
1954 & Susan & 6.1 & 13.2 & 32.5 & David & 6.3 & 17.4 & 37.8 \\
1964 & Susan & 3.6 & 10.3 & 28.6 & Paul & 5.6 & 15.9 & 39.4 \\
1974 & Sarah & 4.9 & 12.3 & 28.0 & Mark & 4.6 & 12.5 & 33.1 \\
1984 & Sarah & 4.1 & 11.0 & 27.3 & James & 4.3 & 11.8 & 32.3 \\
1994 & Emily & 3.4 & 8.6 & 23.8 & James & 4.2 & 11.0 & 28.4 \\
& & & & & & & & \\
\hline & & & & & & &
\end{tabular}

Based on Galbi (2001), table 3 and underlying data. See appendix D for sources.

Before the 19th century, the frequency of the most popular given names in the UK was more stable. Tables 2 and 3 provide evidence on name popularity from late in the 11th century through the early 19th century. From 1300 to 1800 , frequencies of $20 \%, 50 \%$, and $80 \%$ are roughly typical for the most popular name, most popular three names, and most popular ten names for both males and females. The corresponding figures for the late 20th century are much lower-about $4 \%$, $10 \%$, and $25 \%$, respectively. It is important to note that while top name popularities show no overall trend from 1300 to 1800 , the particular names that made up the most popular names from one era to another did change. The causes of changes in individual names goes beyond the scope of this report, but for interested readers the names can be found in the appendix. 
278 Names 50.4 (December 2002)

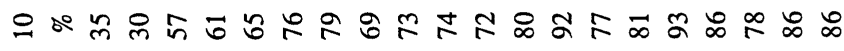
$\stackrel{\circ}{\circ}$

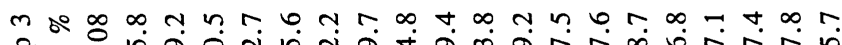

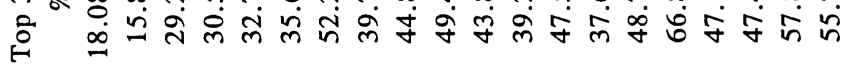

শ

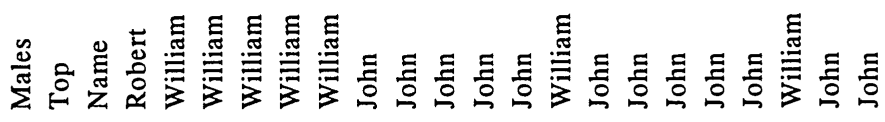

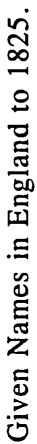

产

$\stackrel{2}{2}$

吕̊ポ

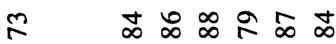

$\stackrel{\circ}{\circ}$

$\overbrace{0}^{m}$

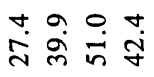

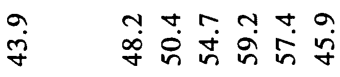

产

离

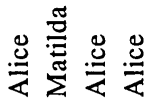

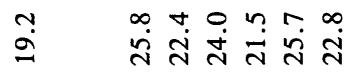

$\stackrel{m}{=} \stackrel{+}{0} \stackrel{\square}{\Xi}$

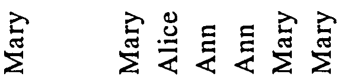




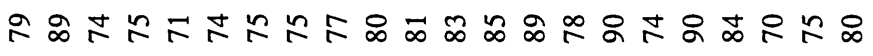

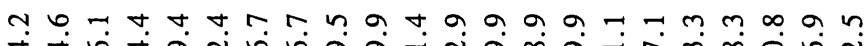

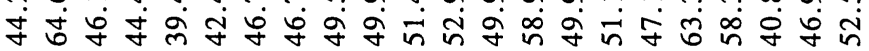

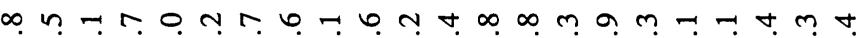
品

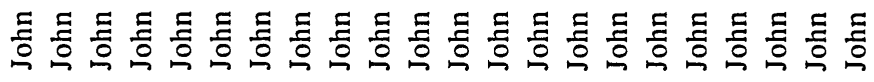

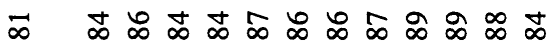

さ $\infty$

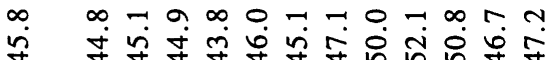

6 in

लं

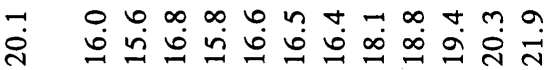

6.

$\stackrel{\infty}{\sim}$

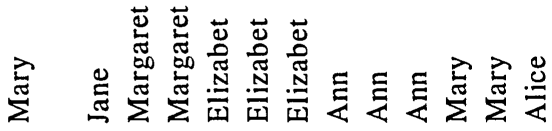

苞

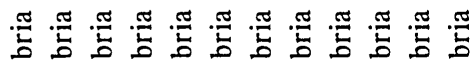

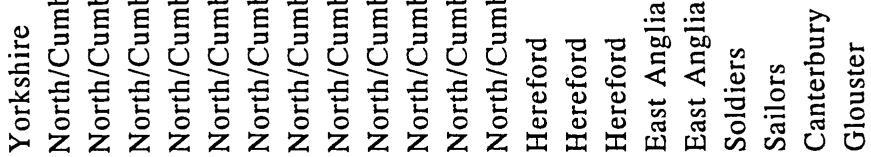

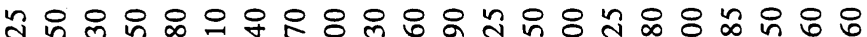

드 


\section{Names 50.4 (December 2002)}

Table 3. Given Names in England, 1570-1700. Combined Frequency of the Three Most Popular Names (in \%).

\begin{tabular}{ccc}
\hline Birth Years & Females & Males \\
$1570-1579$ & 41.0 & 48.5 \\
$1580-1589$ & 36.2 & 47.3 \\
$1590-1599$ & 41.1 & 50.6 \\
$1600-1609$ & 38.2 & 48.8 \\
$1610-1619$ & 38.8 & 49.9 \\
$1620-1629$ & 41.3 & 49.3 \\
$1630-1639$ & 45.1 & 48.5 \\
$1640-1649$ & 46.7 & 49.3 \\
$1650-1659$ & 50.1 & 49.0 \\
$1660-1669$ & 47.5 & 48.0 \\
$1670-1679$ & 50.3 & 50.3 \\
$1680-1689$ & 51.7 & 49.2 \\
$1690-1700$ & 52.1 & 51.2 \\
\hline
\end{tabular}

Source: Smith-Bannister (1997, 150).

Significant social, political, and religious changes in England prior to 1800 seem to have had little effect on the overall distribution of name frequencies. Within a few generations of the Norman Conquest of England, most given names were those brought by the invaders, although there is no evidence that the Norman clergy or court compelled the adoption of Norman names. By about 1250 pre-Conquest names had essentially died out. ${ }^{3}$ Yet by the middle of the 13th century, the distribution of name frequencies was more like that of 1800 than it was of the late 20th century.

\section{Describing Name Frequencies}

The frequencies of the the most popular names follow a general order that can be recognized graphically. Earlier reports on given name frequencies, such as those of Eschel (2001), Tucker $(2001 ; 2002)$, and Galbi (2001) have recognized that associated graphs have a characteristic shape when the logarithm of name popularity is plotted against the logarithm of name popularity rank, which is the same as a graph of name frequencies except that the left axis is labeled in more easily understood units. ${ }^{4}$ The graph typically is close to a straight line. This type of empirical regularity is called a power law and it describes the relative frequency of popularity of names. Hence a power law, in this case, describes a relationship between the popularity of the most frequent name, the three most frequent names, and the ten most frequent names. 
Figure 1. Popularity Distribution of Female Given Names in the UK.

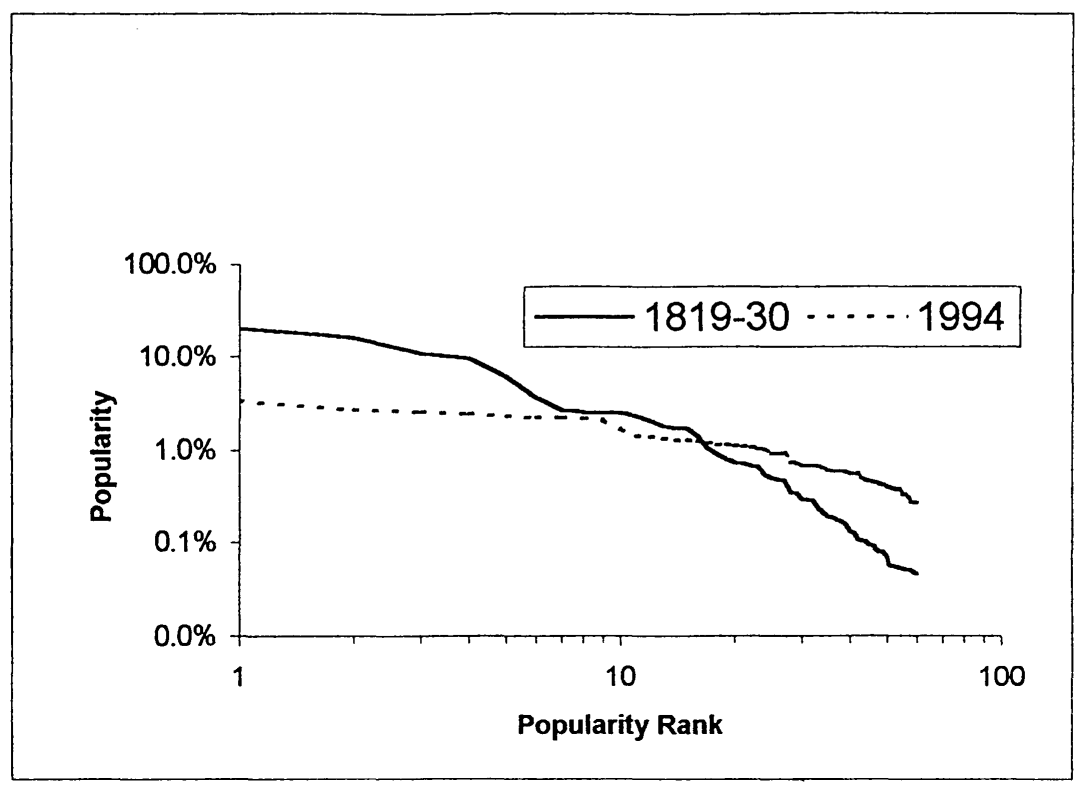

Figure 2. Popularity Distribution of Male Given Names in the UK.

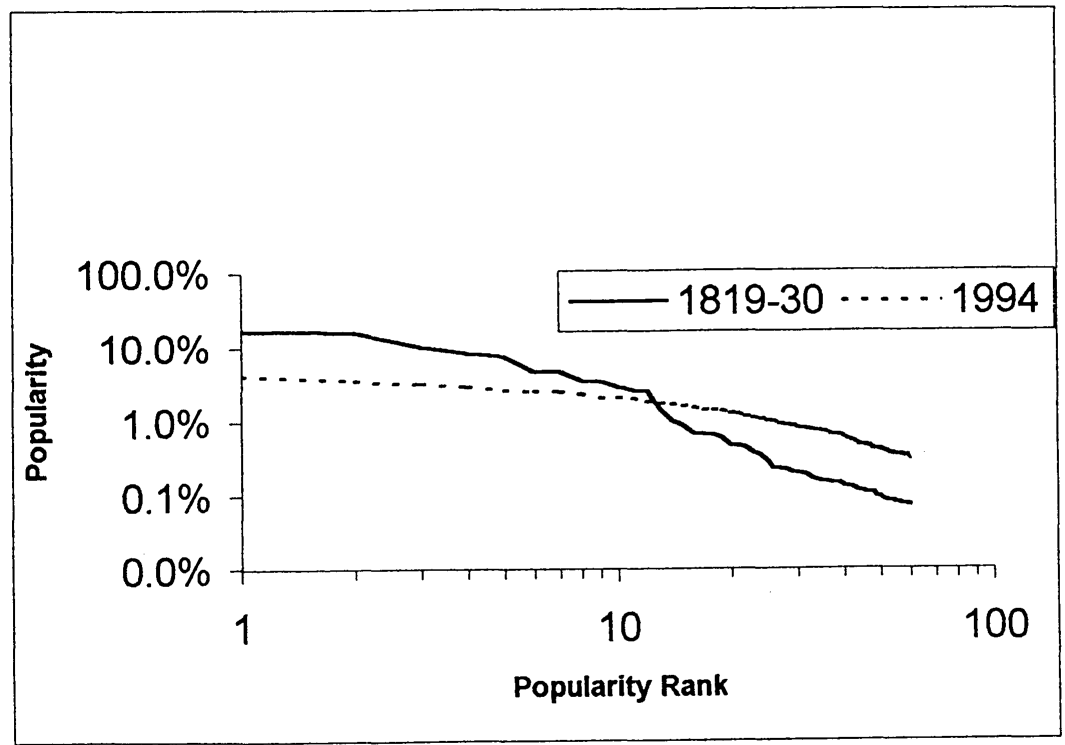




\section{Names 50.4 (December 2002)}

Over the past two hundred years, the change in the popularity of the most frequent names has been associated with a flattening of the power law that best describes the name popularity distribution. Figures 1 and 2 show these graphs for names of females and males born in the UK in 1819-30 and in 1994. For both male and female names the slope of the line approximating the graph has become less negative, indicating that the relative name popularities have become more equal. This change can be interpreted as a reduction in the magnitude of information encoded in the name distribution and an increase in the extent of personalization in naming. (For a discussion of these points see Galbi [2001], Sec. II.B.)

Empirical regularities such as those seen in figures 1 and 2 are in fact prevalent in the information economy. Where persons and organizations are free to create and choose among many collections of symbols instantiated and used in a similar way, the relative popularity of the symbolic artifacts typically follows a power law. The circulation of magazines of similar type throughout the 20th century, the total box office receipts of movies, the popularity of musical groups as measured by gold records produced (Chung and Cox 1994), and the popularity of Internet web sites, measured in users or page views (Adamic and Huberman 2000) have all been shown to follow power laws. Insights into the evolution of such power laws from the study of name changes over time should contribute substantially to a more general understanding of personal preferences, media diversity, information industry structure, and other aspects of the information economy.

\section{Conclusion}

Although recent work on personal given names in England has emphasized name sharing in understanding the frequency distribution of given names (e. g., Smith-Bannister 1997), name sharing practices have little direct relationship to the frequency distribution of names. Naming children after parents, godparents, or ancestors is equally consistent with a high or low popularity of names. Similarly, having names freely chosen; that is, chosen in the absence of norms giving high value to the name of a person in a specific social position, could produce high or low popularity of certain names. The most that can be said for name sharing is that a norm of naming after parents creates additional inertia in name 
popularity. Name popularity and its long-term evolution depend on factors other than name sharing. The evolution of the name frequency distribution over time is a complicated, dynamic system. Such systems can be highly sensitive to a particular factor at one time and totally unaffected by it at another time. Moreover, boundary conditions, such as a small share of naming done in violation of prevailing norms, can contribute to the overall state of the system. ${ }^{5}$

Analysis of long-term trends in personal given names in the UK suggests that significant changes in the information economy occurred in conjunction with the broad social and economic changes brought about by the Industrial Revolution, which began in Britain in the middle of the 18th century. The Industrial Revolution had a number of profound effects on English society. The population of England increased considerably and real economic income per capita increased by about a factor of four from 1300 to 1800 , and by about a factor of 100 from 1800 to $2000 .^{6}$ It is not clear how the level of income might affect the frequency of names since populations of much different sizes show similar naming frequency distributions (Eshel 2001; Galbi 2001, table 4). The Industrial Revolution produced major changes in social networks and the social context of personal activity as well. The influence of these changes on the distribution of given names remains to be examined.

Whether information and communication technologies have created -or will create-a "new economy" is an important public policy issue. These technologies enable persons to interact in new ways that may bring about changes as significant as those associated with the Industrial Revolution. Consider, for example, the creation of knowledge about aggregate patterns of personal given names. Large compilations of name frequencies can be easily shared on the Internet. I have benefited from such sharing of information in writing this article, and I have made much more extensive data on name frequencies available through AGNAMES. ${ }^{7}$ If other scholars use the Internet in similar ways, this subfield of onomastics could develop much more rapidly than it has in the past. The same might be true of many other areas of activity. Analyzing the popularity distribution of personal given names thus offers a particularly rich means for understanding changes in the information economy. 


\section{Names 50.4 (December 2002)}

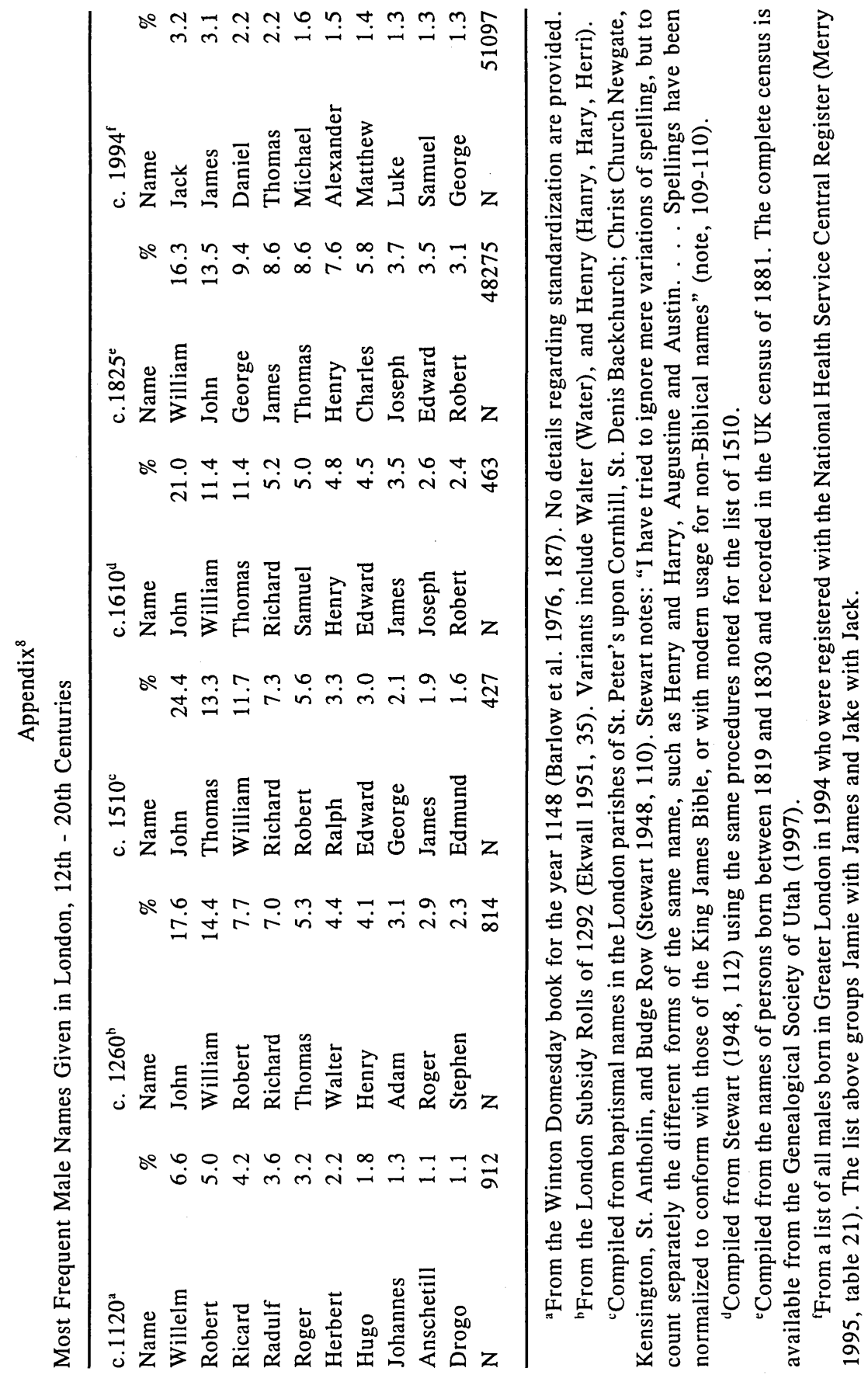


Trends in Given Name Frequencies 285

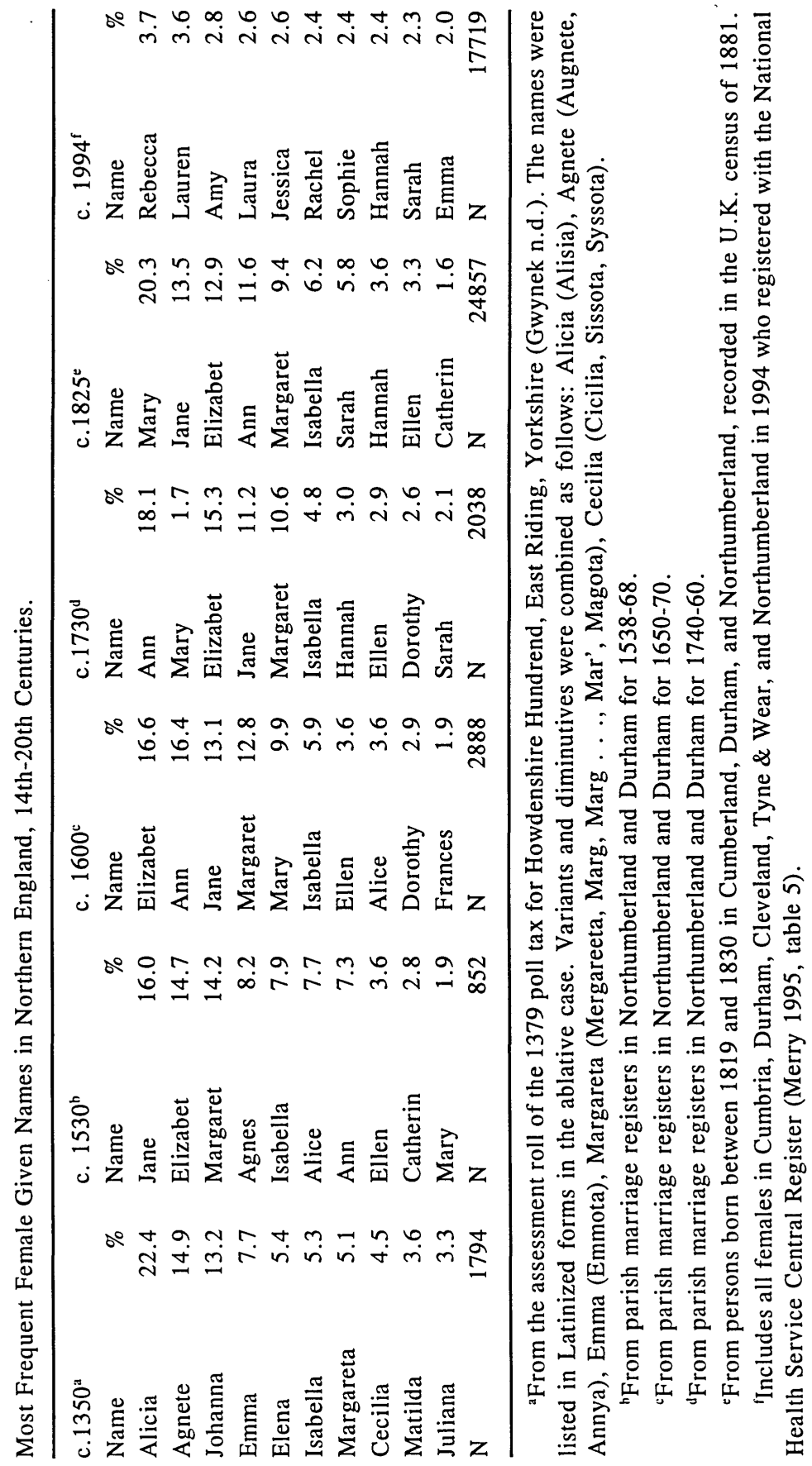




\section{Names 50.4 (December 2002)}

\section{Notes}

The opinions and conclusions expressed here are those of the author and they do not necessarily reflect the views of the Federal Communications Commission, its Commissioners, or any staff other than the author.

1. Available at http://users.erols.com/dgalbi/names/ginap.htm. The principle for coding is to group together names that either sound the same, have the same public meaning, or changed only in the recording process (spelling errors, recording errors, etc.).

2. Among other things, name standardization helps to control for changes in names used as a person grows older, e.g., from Bobby to Bob to Robert. For this reason name standardization is particularly important in analyzing time trends when the data come from naming cohorts constructed by age. This is the case for data presented here on 19th century names.

3. There is no evidence that Norman clergy or royal officials compelled the English to adopt Norman names (Clark 1992, 552, 558-562).

4. This is true because $\log (a / b)=\log (a)+\log (b)$. The logarithm of name frequency differs from the logarithm of name popularity only by an additive factor. Name popularity rank and name frequency rank are of course identical.

5. Gabaix (1999) shows that, when the appearance rate for new cities is not too high, it has no effect on the slope of the power law describing city sizes. If the appearance rate for new cities rises above a certain threshold, then the slope depends on the appearance rate. Cities can be analogized to name types.

6. For population and income statistics for 1700 and earlier, see Mayhew (1995) table I, and Snooks (1995), table 3.5. For current population statistics, see UK National Statistics, Key Population and Vital Statistics, http://www.statistics. gov.uk/statbase/Product.asp? vlnk $=539 \&$ More $=N$. The large changes in the structure of the economy over the past two hundred years make estimating changes in per capita income uncertain. The figure of 100 is an estimate based upon my understanding of the literature on economic history.

7. http://users.erols.com/dgalbi/names/agnames.htm.

8. These listings show the 10 most popular male names in and about London, c. 1120 to 1994 and the 10 most popular female names from north England (Yorkshire, Cumbria, and Northumberland) c. 1350 to 1994. The years given are approximate birth years, estimated relative to the date of compilation and the probable ages of the persons in the compilation. The data come from a variety of sources, which used different and often not explicitly described methods of standardizing and grouping the names. Readers interested in additional name lists should consult Smith-Bannister (1997), appendix C, which lists at decade intervals the 50 most popular male and female names in 40 English parishes from 1538-49 to 1690-1700. Unfortunately Smith-Bannister does not give the frequency of specific names nor the sample sizes. The weights given to individual parishes in each decade sample apparently change, but details are not given. 


\section{References}

Adamic, Lada A., and Bernardo A. Huberman. 2000. "The Nature of Markets in the World Wide Web." Quarterly Journal of Electronic Commerce 1:5-12. Also available online at http://www.parc.xerox. com/istl/groups/iea/abstracts/ECommerce/webmarkets.html.

Barlow, Frank, Martin Biddle, Olof von Feilitzen, and D. J. Keene. 1976. Winchester in the Early Middle Ages: An Edition and Discussion of the Winton Domesday. With contributions from T.J. Brown, H. M. Nixon, and Francis Wormald. Oxford: Clarendon Press.

Chung, Kee H., and Raymond A. K. Cox. 1994. "A Stochastic Model of Superstardom: An Application of the Yule Distribution." The Review of Economics and Statistics 76: 771-75.

Clark, Cecily. 1992. "Onomastics." The Cambridge History of the English Language, 1066-1476. Vol. II. Ed. Richard M. Hogg. Cambridge: Cambridge Univ. Press.

Ekwall, Eilert, ed. 1951. Two Early London Subsidy Rolls. Lund: C. W. K. Gleerup.

Eshel, Amram. 2001. "On the Frequency Distribution of First Names." Names 49: 55-60.

Gabaix, Xavier. 1999. "Zipf's Law for Cities: An Explanation." Quarterly Journal of Economics 114: 739-67.

Galbi, Douglas A. 2001. "A New Account of Personalization and Effective Communication." Available at http://www.galbithink.org or http://www.ssrn.com.

Genealogical Society of Utah, Federation of Family History Societies [GSU-FFHS]. 1881. Census for England and Wales, the Channel Islands and the Isle of Man [computer file, SN: 3643] (Colchester, Essex: The Data Archive [distributor], 29 July 1997).

Mayhew, N. J. 1995. "Population, Money Supply, and the Velocity of Circulation in England, 1300-1700." Economic History Review XLVIII: 238-57.

Merry, Emma. 1995. First Names: The definitive guide to popular names in England and Wales 1944-1994 and in the regions 1994. (With support from Kay Callaghan and Chris Cotton). London: HMSO.

Smith-Bannister, Scott. 1997. Names and Naming Patterns in England, 1538-1700. Oxford: Oxford Univ. Press. 


\section{Names 50.4 (December 2002)}

Snooks, Graeme Donald. 1995. "The Dynamic Role of the Market in the Anglo-Norman Economy and Beyond, 1086-1300." A Commercialising Economy: England 1086 to c.1300. Eds. Richard H. Britnell and Bruce M. S. Campbell. Manchester and New York: Manchester Univ. Press.

Stewart, George R. 1948. Men's Names in Plymouth and Massachusetts in the Seventeenth Century. Berkeley and Los Angeles: Univ. of California Press.

Tucker, D. K. 2001. "Distribution of Forenames, Surnames, and Forename-Surname Pairs in the United States.” Names 49: 69-96. . 2002. "Distribution of Forenames, Surnames, and ForenameSurname Pairs in Canada." Names 50: 105-132. 